\title{
First-line therapy of chronic myeloid leukemia - focus on dasatinib
}

\section{Philip C Amrein}

Hematology-Oncology, Massachusetts General Hospital, Boston, USA
Correspondence: Philip C Amrein Hematology-Oncology, Massachusetts General Hospital, 100 Blossom Street, Boston, MA 02II4, USA

Email pamrein@partners.org
This article was published in the following Dove Press journal:

Blood and Lymphatic Cancer:Targets and Therapy

19 March 2012

Number of times this article has been viewed

\begin{abstract}
Dasatinib is a broad spectrum, ATP-competitive, tyrosine kinase inhibitor with low $\mathrm{nM}$ activity against Bcr-Abl, Src, and other tyrosine kinases. This activity has allowed dasatinib to emerge as one of the most effective agents in the treatment of chronic myeloid leukemia (CML), and dasatinib has become the third drug over the past 10 years to be FDA approved for first line therapy in this setting. This review consists of papers and reports that recount the recent dramatic progress that has been made in the understanding of CML on a molecular basis leading up to the new targeted therapies, especially the use of dasatinib. As with any drug, treatment with dasatinib has risks and side effects, especially pleural effusions and anti-platelet effects, as outlined in recently published studies. Of interest are the reports of alternative doses and schedules that may be able to circumvent some of these side effects without compromising the effectiveness of this drug. This review outlines the mode of action, pharmacology, effectiveness, and safety of dasatinib in the treatment of CML, and by comparing dasatinib directly with the other tyrosine kinase inhibitors effective in CML, it describes the place in therapy that dasatinib currently holds.
\end{abstract}

Keywords: dasatinib, CML, chronic myeloid leukemia

\section{Introduction to natural history and management of CML Classification and prognosis}

The World Health Organization published its updated classification of the hematopoietic neoplasms, including chronic myeloid leukemia (CML) in 2008, and this work represents the opinions of the Consensus Conference of the International Working Group that met in Lyon, France in October 2007. ${ }^{1}$ Accordingly, CML is currently defined as a myeloproliferative neoplasm that is associated with the BCR-ABL1 fusion gene located in the Philadelphia $(\mathrm{Ph})$ chromosome (BCR refers to the "break point cluster" region on chromosome 22, while ABL1 refers to the "abelson" region on chromosome 9, which encodes the tyrosine kinase domain). This leukemia is not common and has a worldwide incidence of only one or two cases per 100,000 population, and in the United States in 2011 there were an estimated 5150 new cases. ${ }^{2}$ Patients frequently present asymptomatically with an elevated white blood cell (WBC) count, but frequently there is associated anemia, fatigue, and splenomegaly. Generally, patients pass from chronic phase (CP) through accelerated phase (AP) to the blast phase (BP) before encountering a fatal event, which is usually bleeding or infection. The accelerated phase of CML has been defined as the presence of $10 \%-19 \%$ blasts in the blood or bone marrow, but the presence of uncontrolled splenomegaly, leukocytosis, thrombocytosis, 
or basophilia greater than $20 \%$ have also been accepted as accelerated phase. ${ }^{1}$ The blast phase of CML is defined by a blast percentage of $20 \%$ or more. In 1993 the median survival of patients newly diagnosed with CML was reported to be 3.8 years if treated with busulfan, 4.7 years if treated with hydroxyurea, and 5.5 years if treated with interferon. ${ }^{3}$ There were no long-term survivors when not treated with a bone marrow transplant. The long-term survival with a bone marrow transplant has improved over the years. In an analysis of recent European outcomes, the 2-year survival with bone marrow transplantation for CML has improved from 54\% in the 1980 s to $74 \%$ in reports from 2000 to $2003 .{ }^{4}$ With the combined use of hydroxyurea, interferon, and cytarabine some progress was reported in 1997 for patients treated outside the bone marrow transplant setting with a median survival of 6 years. ${ }^{5}$ However, with the arrival of imatinib and the other tyrosine kinase inhibitors (TKIs), dasatinib and nilotinib, the prognosis has improved dramatically, such that bone marrow transplantation is now more often used for salvage therapy for patients that have failed TKI therapy.

\section{Development of imatinib}

Brian Druker is credited with the discovery and initial development of imatinib. ${ }^{6}$ In the early 1990s he collaborated with Novartis to explore the possibility of targeting the Bcr-Abl fusion protein, the product of the $B C R-A B L$ gene, which is believed to transform the cells and give rise to the leukemia. Working with many tyrosine kinase inhibitors, they found that agent STI571 (signal transduction inhibitor number 571) effectively inhibited the Bcr-Abl fusion protein, killed the CML cells, and had few off-target effects. The first patients to be treated were in 1998 in a Phase I trial, where safety was established at an effective dose of imatinib of $400 \mathrm{mg}$ once daily. ${ }^{7}$ Subsequent Phase 2 trials established the effectiveness of this drug at this dose and led to the approval on May 10, 2001 of this agent for treatment of CML by the US FDA (United States Food and Drug Administration). ${ }^{8}$ However, the Phase 3 trial named the International Randomized Trial Comparing Interferon to STI571 (IRIS trial) has become the standard database, which has defined the long-term effectiveness and safety of imatinib as first line treatment for patients with CML, as shown by the arm containing imatinib. ${ }^{9}$ Since then, there has been a 5-year analysis ${ }^{10}$ and an 8 -year analysis ${ }^{11}$ of the IRIS trial. The 8 -year analysis showed that $81 \%$ of patients did not progress on imatinib, and the progression to blast phase had decreased to $<3 \%$ per year. The overall survival projections were $90 \%$ at 8 years with a median survival estimated to be well over 15 years. Since then
Novartis (Novartis International AG, Basel, Switzerland) has developed a potentially improved tyrosine kinase inhibitor, nilotinib, with much stronger binding to Bcr-Abl and fewer off target effects. Similarly, Bristol-Myers Squibb (BMS, New York) has developed dasatinib, which also has much stronger binding to Bcr-Abl and apparently fewer off-target effects. Both drugs have now been FDA-approved for initial therapy of CML. ${ }^{12,13}$

\section{Management of CML}

The current management of patients with CML has changed dramatically over the past decade, with new considerations for the initial evaluation, assessment of prognosis, and use of agents, and the expectation that certain "milestones" will be reached at defined time points. The initial evaluation of a CML patient should include history, physical exam, and several blood and bone marrow studies as listed in Table 1. Some still find the Sokal score useful, which provides some indication of prognosis according to: age, spleen size, platelet count, and percentage of blasts in the bone marrow and blood. ${ }^{14}$ Many patients present with a leukocytosis and no urgent need for treatment, such that evaluation can proceed routinely. However, some patients present with a very high WBC, visual changes, profound weakness, and cardiopulmonary compromise. In such urgent cases, it is reasonable to start treatment with hydroxyurea or even leukapheresis. The current recommendation for initial treatment of stable CML patients in chronic phase is to use one of the three FDA-approved tyrosine kinase inhibitors at standard doses. Successful ongoing treatment at the 3-month time point would be indicated by a complete hematological remission (CHR) and a drop below $10 \%$ in the BCR-ABL1 signal in blood by the polymerase chain reaction (PCR) test. ${ }^{15-16}$ Note that the European Leukemia Net (ELN) has set specific guidelines for definitions and expectations of response. ${ }^{17,18}$ According to the ENL, a CHR requires all of the following: $\mathrm{WBC}<10,000 / \mathrm{uL}$, basophils $<5 \%$, no immature myeloid cells other than bands,

Table I Evaluation of the patient with CML

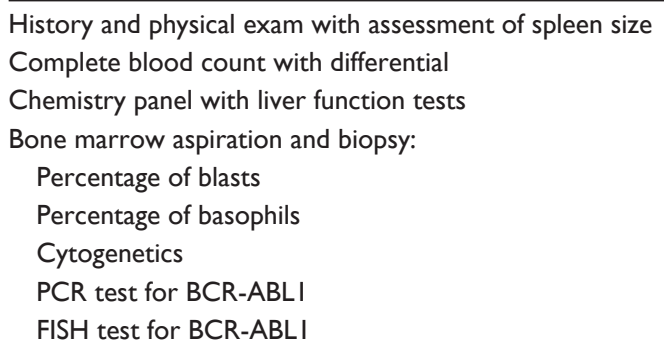

Abbreviations: CML, chronic myeloid leukemia; FISH, fluorescence in situ hybridization; PCR, polymerase chain reaction. 
platelets $<450,000 / \mathrm{uL}$, and a nonpalpable spleen. Also, the ELN defines a complete cytogenetic response (CCyR) as no cells in the bone marrow containing the Philadelphia chromosome $(\mathrm{Ph})$, and a partial cytogenetic response (PCyR) as $1 \%-35 \%$ of marrow cells $\mathrm{Ph}+$. A complete molecular response (CMR) requires no detectable BCR-ABL1 mRNA by PCR in two consecutive tests with a sensitivity $>10^{4}$. Note that the international scale for monitoring $\mathrm{CML}$ patients was created to harmonize the differing methodologies in different laboratories around the world. ${ }^{18}$ After 6 months of TKI therapy, a bone marrow aspiration is recommended and at that point successful therapy would be indicated by at least a PCyR. By 12 months, the patient should have achieved a CCyR and at 18 months the PCR test for BCR-ABL1 should indicate a level $<0.1 \%$. Patients in the IRIS trial who have achieved a CCyR by 18 months have had a very low frequency of progression and may have an indefinite disease-free survival. ${ }^{11}$ Patients not achieving a goal at the specified time point, or showing signs of progression, should be re-tested within 1 month. If still not achieving the goal, an ABL kinase domain mutational analysis should be performed on blood or bone marrow cells to determine whether one of the known ABL kinase mutations is responsible for the resistant disease. As listed in Table 2, certain ABL mutations may be less resistant to one TKI or the other, and this may influence treatment decisions. ${ }^{19}$ If the T315I mutation is encountered, neither nilotinib nor dasatinb would be effective, and such patients should be treated in a clinical trial or undergo cytoreduction with conventional chemotherapy followed by an allogeneic bone marrow transplant. Of note is a report of initial effectiveness of the experimental agent ponatinib in the presence of the T315I ABL mutation. ${ }^{20}$

\section{Overview of pharmacology of dasatinib and rationale for use in first line treatment of CML}

Dasatinib is a broad spectrum ATP (adenosine triphosphate)competitive inhibitor of five oncogenic tyrosine kinases: Bcr-Abl, Src, c-Kit, platelet-derived growth factor (PDGF)

Table 2 Therapeutic choice according to ABL kinase mutation ${ }^{19}$

\begin{tabular}{lll}
\hline Mutations & $\begin{array}{l}\text { Drug } \\
\text { resistance }\end{array}$ & $\begin{array}{l}\text { Consider choice } \\
\text { of therapy }\end{array}$ \\
\hline V299L, T3I5A, F3 I7L/V/I/C & Dasatinib & Nilotinib \\
Y253H, E255K/V, F359V/C/I & Nilotinib & Dasatinib \\
T315I & Both drugs & Clinical trial \\
\hline
\end{tabular}

Note: "ABL" is that portion of the BCR-ABL fusion gene which contains the tyrosine kinase domain. receptor $\mathrm{G}$, and ephrin receptor kinases. ${ }^{21-23}$ The IC50 for Bcr-Abl is approximately $3 \mathrm{nM}$, which indicates that it is at least 300 times more potent than imatinib for this target. In vitro experiments showed that dasatinib killed a wide spectrum of Bcr-Abl dependent leukemia cell lines, although not those containing the T315I mutation. In the xenograph murine model using K562 CML cells resistant to imatinib, treatment with dasatinib was effective in these animals. ${ }^{24,25}$ In human studies, oral dosing showed maximum plasma concentrations at 0.5 hours and 6 hours with a mean terminal half life of 3-5 hours. ${ }^{26}$ Absorption from the G-I tract is reduced by high $\mathrm{pH}$ of the stomach contents but not, in general, by the presence of food. Dasatinib is extensively distributed in the extravascular space, even the central nervous system. It is extensively metabolized in the liver by the P450 enzyme CYP3A4. It is a weak time-dependent inhibitor of CYP3A4 but does not inhibit or induce the other CYP enzymes at relevant concentrations. Approximately $85 \%$ of orally ingested dasatinib is eliminated in the feces, while $4 \%$ is eliminated in the urine after 10 days.

Safety and efficacy of dasatinib in CML and Ph+ ALL patients was determined in several Phase 1 and Phase 2 trials. ${ }^{27-29}$ The Phase 3 randomized trial comparing four schedules of dasatinib in chronic phase CML patients, either resistant to or intolerant of imatinib, defined the optimum schedule for dasatinib to be $100 \mathrm{mg}$ once daily. This schedule was compared to $50 \mathrm{mg}$ BID, $70 \mathrm{mg}$ BID, and $140 \mathrm{mg}$ once daily of dasatinib. ${ }^{30}$ In this study, after taking $100 \mathrm{mg}$ once daily for 6 months, $90 \%$ of patients achieved a complete hematologic remission, and $41 \%$ achieved a complete cytogenetic remission. Adverse reactions of all grades in this CML study for $100 \mathrm{mg}$ once daily were as follows: $24 \%$ fluid retention (10\% pleural effusions), $23 \%$ diarrhea, and $10 \%$ hemorrhage. Myelosuppression was frequently severe in the first month, but much of this was thought to be due to the leukemia and also due to mature cells containing the BCR-ABL1 fusion gene. With remission, patients could be continued on dasatinib without significant myelosuppression. Prolongation of the QTc interval as measured by electrocardiogram (EKG) was seen, but the mean change from baseline was only 3-6 ms. Fewer than $1 \%$ of patients had a prolongation of the QTc $>500 \mathrm{~ms}$.

The effectiveness of dasatinib relative to imatinib was tested in a randomized trial in 519 patients newly diagnosed with CML (the DASISION trial). ${ }^{31}$ In this trial, after a minimum follow-up of 12 months, the complete cytogenetic response in the dasatinib arm was $77 \%$ compared to $66 \%$ in the imatinib arm (Table 3), and that was statistically 
Table 3 Effectiveness of dasatinib compared to imatinib at 12 months (DASISION trial) ${ }^{31}$

\begin{tabular}{llll}
\hline Response & $\begin{array}{l}\text { Dasatinib } \\
\text { (\%) }\end{array}$ & $\begin{array}{l}\text { Imatinib } \\
\text { (\%) }\end{array}$ & Significance \\
\hline $\begin{array}{l}\text { Complete cytogenetic } \\
\text { response }\end{array}$ & 77 & 66 & $P=0.007$ \\
$\begin{array}{l}\text { Major molecular response } \\
\text { Progression to AP or BP }\end{array}$ & 46 & 28 & $P=0.000$ I \\
\hline
\end{tabular}

Abbreviations: AP, accelerated phase; BP, blast phase; NA, not available. Note: Reproduced with the permission of the publisher from Kantarjian $\mathrm{H}$, Shah NP, Hochhaus A, et al. Dasatinib versus imatinib in newly diagnosed chronic-phase chronic myeloid leukemia. N EnglJ Med. 2010;362(24):2260-2270. (C) Massachusetts Medical Society.

significant $(P=0.007)$. The rate of progression to accelerated phase or blast phase of CML was $1.9 \%$ for dasatinib and $3.5 \%$ for imatinib. The safety data for dasatinib in this trial compared well with that for imatinib, but tests of significance were not provided (Table 4). This trial led to the FDA approval of dasatinib as first-line therapy for CML patients in October 2010. ${ }^{12}$ Of note, also at this time, nilotinib was compared to imatinib in a randomized trial of 846 patients with newly diagnosed CML (the ENESTnd trial, see Table 5) and, similarly, was found to be slightly more effective than imatinib with a complete cytogenetic response of $78 \%-80 \%$ at 12 months compared to $65 \%$ for imatinib ${ }^{32}$, and this led to

Table 4 Toxicities attributed to the administered tyrosine kinase inhibitor (DASISION trial) ${ }^{31}$

\begin{tabular}{|c|c|c|c|}
\hline Category & Toxicity & $\begin{array}{l}\begin{array}{l}\text { Dasatinib } \\
(\mathrm{N}=258)\end{array} \\
\begin{array}{l}\text { Frequency } \\
(\%)\end{array}\end{array}$ & $\begin{array}{l}\text { Imatinib* } \\
(\mathbf{N}=\mathbf{2 5 8}) \\
\text { Frequency } \\
(\%)\end{array}$ \\
\hline Hematopoietic & Neutropenia & 21 & 20 \\
\hline (Grades $3+4)$ & Thrombocytopenia & 19 & 10 \\
\hline Non-hematopoietic & Fluid retention & 19 & 42 \\
\hline \multirow[t]{14}{*}{ (all grades) } & Pleural effusion & 10 & 0 \\
\hline & Diarrhea & 17 & 17 \\
\hline & Headache & 12 & 10 \\
\hline & Rash & II & 17 \\
\hline & Musculoskeletal pain & II & 14 \\
\hline & Nausea & 8 & 20 \\
\hline & Fatigue & 8 & 10 \\
\hline & Myalgias & 6 & 12 \\
\hline & Vomiting & 5 & 10 \\
\hline & Bleeding & 5 & 5 \\
\hline & Muscle & 4 & 17 \\
\hline & inflammation & & \\
\hline & Musculoskeletal pain & II & 14 \\
\hline & $\mathrm{QTc}>500 \mathrm{~ms}$ & 0.4 & 0.4 \\
\hline
\end{tabular}

Notes: "No tests of statistical significance were provided comparing the two treatment groups. Reproduced with the permission of the publisher from Kantarjian $\mathrm{H}$, Shah NP, Hochhaus A, et al. Dasatinib versus imatinib in newly diagnosed chronic-phase chronic myeloid leukemia. N Engl J Med. 20I0;362(24):2260-2270. (c) Massachusetts Medical Society.
Table 5 Effectiveness of nilotinib compared to imatinib at 12 months (ENESTnd trial) ${ }^{32}$

\begin{tabular}{llll}
\hline Response & $\begin{array}{l}\text { Nilotinib } \\
(\%)\end{array}$ & $\begin{array}{l}\text { Imatinib } \\
\text { (\%) }\end{array}$ & Significance \\
\hline $\begin{array}{l}\text { Complete cytogenetic } \\
\text { response }\end{array}$ & $78-80$ & 65 & $P=0.001$ \\
$\begin{array}{l}\text { Major molecular response } \\
\text { Progression to AP or BP }\end{array}$ & $\begin{array}{l}43-44 \\
<1\end{array}$ & $\begin{array}{l}22 \\
4\end{array}$ & $\begin{array}{l}P=0.001 \\
\text { NA }\end{array}$ \\
\hline
\end{tabular}

Abbreviations: AP, accelerated phase; BP, blast phase; NA, not available.

the FDA approval of nilotinib for first-line therapy in CML patients. ${ }^{13}$ In both trials, the rate of decline of the BCR-ABL mRNA transcript appeared to be faster with the newer agents than with imatinib.

The overall rationale for use of dasatinib as first-line therapy for newly diagnosed patients with CML in chronic phase would rest with its apparent improved efficacy over imatinib to achieve a complete cytogenetic response and its slight advantage in adverse events, although pleural effusions are more frequent with dasatinib than with imatinib, and may result in temporary discontinuation of dasatinib. ${ }^{31}$ Nilotinib also has demonstrated improved efficacy over imatinib to achieve complete cytogenetic responses, and it has a slight advantage over imatinib in adverse events, although the greater frequency of rashes and abnormal liver tests is noted. ${ }^{32}$ Neither dasatinib nor nilotinib has the long-term, 8- to 10-year, track record of success that imatinib has enjoyed, but there is no reason yet to suspect that these newer agents will be inferior.

There has emerged an additional rationale to using dasatinib (or nilotinib) instead of imatinib as first-line therapy for CML, and that relates to the possibility of stopping all therapy in some patients that achieve a complete molecular remission (CMR) lasting at least 2 years. There were five studies presented at the American Society of Hematology meeting in December 2011 demonstrating that stopping TKI therapy was reasonable, safe, and a successful strategy for a subset of patients with CMR. ${ }^{33-37}$ This strategy would seem to favor patients with longer and more profound molecular remissions, and this, perhaps, would be more likely with dasatinib (or nilotinib). A French group stopped imatinib treatment in 100 patients with CML who achieved a CMR for at least 2 years, as determined by the RT-PCR technique for the BCR-ABL1 transcript. ${ }^{33}$ By 7 months, 58 had relapsed and three more relapsed by 22 months, but none relapsed after that, leaving 39 of 100 patients off therapy without relapse for a median of 30 months. Those who relapsed generally could be returned to CMR by restarting imatinib. A study from Korea showed that among 20 patients with CML in 
CMR from imatinib for 5 years (median), could stop their treatment. ${ }^{34}$ Their follow-up after stopping it was short, only 7 months, but only two of the 20 patients have shown any sign of molecular progression. A study from Japan retrospectively analyzed 43 patients who stopped imatinib after at least 6 months of achieving a CMR. ${ }^{35}$ In this study $47 \%$ of patients remained in CMR, however, this result differed for patients in CMR for $>24$ months versus those in CMR for $<24$ months, $78 \%$ vs $15 \%(P=0.0002)$. In this study a higher imatinib dose intensity appeared to correlate with more patients experiencing a prolonged CMR. A second Japanese study was presented analyzing 30 patients for whom imatinib was stopped after at least 24 months of sustained CMR, but with a median of 55.5 months in sustained CMR. ${ }^{36}$ Their follow-up is short, median only 5 months, but it appears that 14 of the 30 patients have no evidence for progression by RT-PCR for the BCR-ABL1 transcript. A comment is made that all relapsed patients have responded to re-treatment with TKI therapy. A fifth study reported from France analyzed 16 patients treated with dasatinib (nine) or nilotinib (seven) for intolerance of imatinib or CML resistance to imatinib. ${ }^{37}$ The TKI was stopped after at least 21 months of sustained undetectable molecular residual disease (UMRD), and all patients have been followed for at least 6 months since discontinuation of therapy. A major molecular response (MMR) was lost in five of the 16 patients, all before the 6 month follow-up visit. The remainder, eleven of 16 patients, remained off therapy for up to 20 months. All patients retreated with TKI therapy for progression have returned rapidly to MMR and UMRD status. While the prospect of being able to stop all TKI therapy after several years of CMR is very encouraging, because the duration of observation of these patients is very short, stopping therapy for such patients should not be considered standard practice at this time, and stopping therapy should be reserved only for patients in the clinical trials testing this concept.

\section{Efficacy studies for dasatinib, including comparison studies}

The current measures of effectiveness for the treatment of CML in most recent trials are the following: the percentage of patients achieving CCyR, MMR, CMR, the percentage of patients without progression (EFS), and the percentage of patients not progressing to AP or BP. (Note that EFS includes patients not progressing to AP or BP as well as patients not losing their complete hematologic response or major cytogenetic response.) It is clear from the IRIS trial that improvements in disease status continue from the third month of therapy through 18 months of therapy and beyond. ${ }^{9-11}$ A reasonable time point for comparisons is the 12-month time point. Also, the achievement of CCyR and MMR at 6 months and 12 months clearly predicts excellent EFS and freedom from progression to AP and BP in subsequent years.

Consider some details from the IRIS trial that have been published over the years. ${ }^{9,10}$ In this trial 1106 patients with newly diagnosed CML in chronic phase were randomly assigned to receive either imatinib or interferon plus low-dose cytarabine. Among the 553 patients treated with imatinib, at 12 months $68 \%$ of patients had achieved a CCyR and 39\% had achieved a MMR. In a recently published analysis by Hughes et al, the achievement of MMR at 12 months was shown to significantly predict an excellent outcome with an EFS at 7 years of $91 \%$ and a freedom from AP or BP of $99 \%$ compared to the remainder of the patients with an EFS of $79 \%(P=0.001)$ and a freedom from AP or BP of 90\% $(P=0.0004) .{ }^{38}$ An additional study by Hochhaus et al analyzing data from the 6-year time point showed that CCyR at 6 months was predictive of an excellent outcome with an EFS of $91 \%$ for that group, compared to an EFS of $85 \%$ for those with a partial cytogenetic response at 6 months, and an EFS of $58 \%$ for those with minor or minimal cytogenetic response at 6 months. ${ }^{39}$ Similarly, the freedom from AP and BP was $97 \%$ for the CCyR group compared to $94 \%$ for those with a partial cytogenetic response at 6 months, and $85 \%$ for those with a minor or minimal cytogenetic response at 6 months. From these data it seems reasonable to consider that the best therapy will be that which achieves the deepest CCyR or MMR in the first year of treatment.

The two recently published studies comparing dasatinib to imatinib (the DASISION trial) and nilotinib to imatinib (the ENESTnd trial) provide detailed information regarding measures of effectiveness for these drugs. ${ }^{31,32}$ At 12 months, for patients treated with dasatinib with the current standard dose of $100 \mathrm{mg}$ daily, the CCyR was $77 \%$, the MMR was $46 \%$, and the progression to accelerated phase or blast phase was $1.9 \%$ (see Table 3 ). For imatinib, at 12 months the CCyR was $66 \%(P=0.007)$, MMR was $28 \%(P=0.0001)$, and the rate of progression to AP or BP was 3.5\%. The trial comparing nilotinib to imatinib utilized two doses of nilotinib, $300 \mathrm{mg}$ twice daily and $400 \mathrm{mg}$ twice daily. ${ }^{32}$ Both doses had similar results somewhat improved over those seen with imatinib (Table 5). At 12 months, for patients treated with nilotinib, the rates of CCyR were $78 \%-80 \%$, the rates of MMR were $43 \%-44 \%$, and the rate of progression to AP or BP at $1-2$ years was $<1 \%$. For imatinib in this study at 12 months, the 
rate of CCyR was $65 \%(P<0.001)$, the rate of MMR was $22 \%(P<0.001)$, and the rate of progression to AP or BP was $4 \%$ for the imatinib group. In both trials there was a similar improvement in CCyR and MMR for the newer TKI compared to imatinib. There has been a recent 24-month update of the DASISION trial showing continued improvement for CCyR for both drugs with a smaller advantage for dasatinib $(86 \%$ CCyR) compared to imatinib ( $83 \%$ CCyR) ${ }^{40}$ It is hoped that with the deeper responses with these newer agents there will be a sustained improvement in EFS, and freedom from AP and BP, as would be predicted from the IRIS trial.

An additional study of dasatinib as front-line therapy in 87 newly diagnosed CML patients has recently been reported. ${ }^{41}$ In this study, at 12 months $95 \%$ achieved a CCyR, and $73 \%$ achieved a MMR. A CMR was achieved in $67 \%$ of patients, and with a median follow-up of 29 months the EFS is $93 \%$ with no patients progressing to AP or BP.

\section{Safety and tolerability}

The safety and tolerability of dasatinib as first-line therapy in CML has been reviewed in detail in the DASISION trial. ${ }^{31}$ The dose of dasatinib in this study was $100 \mathrm{mg}$ once daily by mouth, and toxicities compared favorably to those in the imatinib arm (see Table 4). Hematologic toxicities in the form of myelosuppression were common in the first 4 months, a phenomenon common and expected for the TKIs, since the mature cells in the marrow and in the blood initially contain the BCR-ABL1 fusion gene, the target of the drug. For dasatinib (as with imatinib), Grade 3 and 4 neutropenia occurred in 21\% of patients (by the NCI Common Terminology Criteria for Adverse Events Grading System version 3.0, National Cancer Institute, Bethesda, MD). Grade 3 and 4 thrombocytopenia occurred in $19 \%$ of patients. Among the 258 patients treated with dasatinib, only four patients discontinued treatment due to myelosuppression. The most common non-hematologic toxicity was fluid retention. Overall, fewer patients in the dasatinib group experienced this adverse event than those in the imatinib group, $19 \%$ compared to $42 \%$, nearly all Grades 1 and 2, although no test of statistical significance was provided. However, $10 \%$ in the dasatinib group experienced a Grade 1 or 2 pleural effusion (no higher grade pleural effusions), while there were no pleural effusions recorded for the imatinib group. The pleural effusions were treated fairly routinely with medications and dose-modification for the dasatinib until resolution. Specifically, for these 26 patients, three underwent thoracentesis, 12 received diuretics, and seven received corticosteroids. Effectiveness of therapy was not affected, since by 12 months 24 of these 26 patients had achieved a complete cytogenetic response. Other toxicities were similarly uncommon for both drugs in this trial, and generally they were Grade 1 or 2 .

In vitro studies have demonstrated a mild anti-platelet effect for dasatinib, and there has been concern in clinical trials that there may be an observable increase in bleeding among patients treated with dasatinib. ${ }^{42}$ However, in the DASISION trial bleeding events in the dasatinib group occurred with the same frequency (5\%) as in the imatinib group. ${ }^{31}$ Similarly, the QTc interval, as measured by the EKG, can be prolonged with many of the TKIs, but in this trial, the median change from baseline for dasatinib was somewhat less than that for imatinib, $3.0 \mathrm{~ms}$ vs $8.2 \mathrm{~ms}$, and only one patient in each group had a QTc recorded over $500 \mathrm{~ms}^{31}$ In both arms of this trial there was a death due to myocardial infarction possibly related to the study drug. Overall, $5 \%$ in the dasatinib group and $4.3 \%$ in the imatinib group discontinued treatment due to drug adverse events, however, when other causes for discontinuation of treatment were considered (such as disease progression) overall, 15.5\% of patients in the dasatinib arm and $18.6 \%$ of patients in the imatinib arm discontinued treatment.

On October 11, 2011 the US FDA announced in a safety communication that dasatinib may increase the risk of a rare but serious condition, pulmonary arterial hypertension $(\mathrm{PAH})^{12}$ This warning is based on 12 documented cases among 32,882 patients known to have been treated with dasatinib at the time. The incidence is thought to be $<1 \%$ of users, and this warning is now included in the dasatinib label. Signs of PAH include dyspnea, fatigue, hypoxia, and fluid retention. The condition may be reversible on discontinuation of dasatinib. If $\mathrm{PAH}$ is confirmed by right heart catheterization, it is recommended that dasatinib be permanently discontinued.

\section{Patient-focused perspectives, such as quality of life, patient satisfaction/ acceptability, adherence, and uptake}

Patient acceptance of a new medication is not only based on how well the drug controls the disease along with side effects, but also on the convenience and ease of taking the drug. Dasatinib has some advantages and disadvantages in ease of administration. The once-daily dosing schedule with the $100 \mathrm{mg}$ tablet is certainly easier to follow than the more frequent dosing schedules of other medications. The fact that it can be taken with food also allows dosing at breakfast or other convenient times with meals or snacks. Not so convenient is the poor absorption with antacids, and, of course, many patients are on antacids for heartburn and stomach ulcer symptoms. 
While there has been little seen of QTc prolongation with dasatinib, it is recommended that an EKG be taken from time to time, especially if other drugs are being taken which prolong the QTc interval. Dasatinib also affects the P450 pathway, such that other drugs metabolized via this pathway need to be checked and monitored or even discontinued. ${ }^{26}$ There has been concern regarding the incidence of pleural effusions leading to dyspnea and pleuritic chest pain in a subset of patients. While alarming at one level, the problem can be routinely treated with a short course of corticosteroids, diuretics, and holding the dasatinib. ${ }^{31}$ Analysis of altered schedules for dasatinib has produced interesting results. While it was feared that lower doses of dasatinib or missed days of treatment might reduce the effectiveness of dasatinib, this has not occurred in the trials that have analyzed these schedule alterations. In one retrospective study of 31 patients with CML treated with dasatinib due to resistance or intolerance of imatinib, interruptions of treatment from continuous daily dosing were instituted due to toxicity. ${ }^{43}$ Patients were subsequently treated on 3-5 days per week (2-4 days off per week). Surprisingly, it was found that effectiveness was not compromised, and, in fact, in 12 patients an improved response level was achieved compared to that on the continuous daily dosing schedule. Likewise, a trial analyzing the effects of reducing the daily dose of dasatinib from $100 \mathrm{mg}$ daily to $50 \mathrm{mg}$ daily, also resulted in no change in effectiveness. ${ }^{44}$ In this trial nine patients failing imatinib therapy were treated with $50 \mathrm{mg}$ dasatinib daily. All nine patients achieved a complete cytogenetic response within 3 months, and this was sustained through 12 months. At the 18-month time point MMR was achieved for eight of the nine, and one patient achieved a CMR. In the original dose-escalation Phase 1 trial of dasatinib in patients with CML resistant to imatinib, nearly all of the patients receiving dasatinib at $50 \mathrm{mg}$ daily or higher achieved a complete hematologic response. ${ }^{27}$ This wide range of apparent effective doses and schedules allows a considerable convenience for most patients, who typically may miss a few days a month, or need to reduce the dose due to side effects. Of course, these alterations cannot be considered standard treatment at this time due to the small numbers of patients analyzed and the short follow-up.

\section{Conclusion, place in therapy}

Dasatinib represents an advance in therapy over imatinib for patients with CML. Although the experience with dasatinib is many years less than that for imatinib, there seems to be no reason to expect less effectiveness or even additional concerns regarding toxicities. The same can be said for the other relatively new TKI, nilotinib, which appears also to be somewhat more effective and less toxic compared to imatinib. There are important differences between dasatinib and nilotinib in side effects and risks, which will result in the preferred use of one over the other for the individual patient. For patients who need to take antacid medications, nilotinib may be preferred, since stomach $\mathrm{pH}$ is not critical for absorption of this drug. On the other hand, dasatinib can be taken with food, and nilotinib absorption is reduced with food, leading to the requirement that nilotinib ingestion must be scheduled twice daily 1 hour before and 2 hours after meals, such as 10.00 am and $10.00 \mathrm{pm}$. The small subset of patients with significant pleural effusions on dasatinib may find switching to nilotinib preferable to altering the schedule of dasatinb. There is a "black box" warning for nilotinib due to the risk of sudden death from prolonged QT interval with its use. Although this adverse event was a rare event in clinical trials, and dasatinib also prolongs the QT interval, there is no "black box" warning with dasatinib. Patients on drugs that affect the QT interval might better be treated with dasatinib than with nilotinib. Future clinical trials will better define which patients are best treated with dasatinib, but the overall outlook for patients with CML is very encouraging.

\section{Disclosure}

The author declares no conflicts of interest in this work.

\section{References}

1. Vardiman JW, Melo JV, Baccarani M, Thiele J. Chronic myelogenous leukemia, BCR-ABL1 positive. In: Swerdlow SH, Campo E, Harris $\mathrm{NL}$, et al, editors. WHO Classification of Tumors of Haematopoietic and Lymphoid Tissues. 4th ed. Lyon, WHO Press; 2008:32-37.

2. Siegel R, Ward E, Brawley O, Jemal A. Cancer statistics, 2011: the impact of eliminating socioeconomic and racial disparities on premature cancer deaths. CA: Cancer J Clin. 2011;61(4):212-236.

3. Hehlmann R, Heimpel H, Hasford J, et al. Randomized comparison of interferon-alpha with busulfan and hydroxyurea in chronic myelogenous leukemia. The German CML Study Group. Blood. 1994;84(12): 4064-4077.

4. Gratwohl A, Brand R, Apperley J, et al. Allogeneic hematopoietic stem cell transplantation for chronic myeloid leukemia in Europe 2006: transplant activity, long-term data and current results: an analysis by the Chronic Leukemia Working Party of the European Group for Blood and Marrow Transplantation (EBMT). Haematologica. 2006;91(4):513-521.

5. Guilhot $\mathrm{F}$, Chastang $\mathrm{C}$, Michallet $\mathrm{M}$, et al. Interferon alfa-2b combined with cytarabine versus interferon alone in chronic myelogenous leukemia. French Chronic Myeloid Leukemia Study Group. $N$ Engl J Med. 1997;337(4):223-229.

6. Druker BJ, Lydon NB. Lessons learned from the development of an abl tyrosine kinase inhibitor for chronic myelogenous leukemia. J Clin Invest. 2000;105(1):3-7.

7. Druker BJ, Talpaz M, Resta DJ, et al. Efficacy and safety of a specific inhibitor of the BCR-ABL tyrosine kinase in chronic myeloid leukemia. N Engl J Med. 2001;344(14):1031-1037. 
8. Cohen MH, Williams G, Johnson JR, et al. Approval summary for imatinib mesylate capsules in the treatment of chronic myelogenous leukemia. Clin Cancer Res. 2002;8(5):935-942.

9. O'Brien SG, Guilhot F, Larson RA, et al for IRIS Investigators. Imatinib compared with interferon and low-dose cytarabine for newly diagnosed chronic-phase chronic myeloid leukemia. $N$ Engl J Med. 2003;348(11):994-1004.

10. Druker BJ, Guilhot F, O’Brien SG, et al for IRIS Investigators. Five-year follow-up of patients receiving imatinib for chronic myeloid leukemia. N Engl J Med. 2006;355(23):2408-2417.

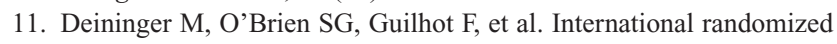
study of interferon vs STI571 (IRIS) 8-year follow up: sustained survival and low risk for progression or events in patients with newly diagnosed chronic myeloid leukemia in chronic phase (CML-CP) treated with imatinib. Blood. (ASH Annual Meeting Abstracts) 2009;114(22): Abst 1126.

12. fda.gov [homepage on the Internet]. US Food and Drug Association. Available from: http://www.fda.gov, search for "dasatinib." Accessed February 20, 2012.

13. fda.gov [homepage on the Internet]. MD: US Food and Drug Association. Available from: http://www.fda.gov, search for "nilotinib." Accessed February 20, 2012.

14. Sokal JE, Baccarani M, Russo D, Tura S. Staging and prognosis in chronic myelogenous leukemia. Semin Hematol. 1988;25(1):49-61.

15. Hanfstein B, Muller MC, Erben P, et al. Molecular and cytogenetic response after 3 months of imatinib treatment is predictive for the risk of disease progression and death in newly diagnosed chronic myeloid leukemia patients - a follow-up analysis of the German CML Study IV. Blood. (ASH Annual Meeting Abstracts) 2011;118(21):Abst 783.

16. Milojkovic D, Ibrahim AR, Foroni L, et al. Assessment of BCR-ABL1 transcript levels at 3 months is the only requirement for predicting outcome for patients with chronic myeloid leukemia treated with imatinib. Blood. (ASH Annual Meeting Abstracts) 2011;118(21):Abst 1680.

17. Baccarani M, Cortes J, Pane F, et al for European LeukemiaNet. Chronic myeloid leukemia: an update of concepts and management recommendations of European LeukemiaNet. J Clin Oncol. 2009;27(35): 6041-6051.

18. Hughes T, Deininger M, Hochhaus A, et al. Monitoring CML patients responding to treatment with tyrosine kinase inhibitors: review and recommendations of harmonizing current methodology for detecting BCR-ABL transcripts and kinase domain mutations and for expressing results. Blood. 2006;108(1):28-37.

19. Branford S, Melo JV, Hughes TP. Selecting optimal second-line tyrosine kinase inhibitor therapy for chronic myeloid leukemia patients after imatinib failure: does the BCR-ABL mutation status really matter? Blood. 2009;114(27):5426-5435.

20. Cortes JE, Kim D-W, Pinilla-Ibarz J, et al for The PACE Study Group. Initial findings from the PACE trial: a pivotal phase 2 study of ponatinib in patients with CML and $\mathrm{Ph}+\mathrm{ALL}$ resistant or intolerant to dasatinib or nilotinib, or with the T315I mutation. Blood. (ASH Annual Meeting Abstracts) 2011;118(21):Abst 109.

21. O'Hare T, Walters DK, Stoffregen EP, et al. In vitro activity of Bcr-Abl inhibitors AMN107 and BMS-354825 against clinically relevant imatinib-resistant $\mathrm{Abl}$ kinase domain mutants. Cancer Res. 2005;65(11):4500-4505.

22. Schittenhelm MM, Shiraga S, Schroeder A, et al. Dasatinib (BMS354825), a dual SRC/ABL kinase inhibitor, inhibits the kinase activity of wild-type, juxtamembrane, and activation loop mutant KIT isoforms associated with human malignancies. Cancer Res. 2006;66(1):473-481.

23. Li J, Rix U, Fang B, et al. A chemical and phosphoproteomic characterization of dasatinib action in lung cancer. Nat Chem Biol. 2010;6(4): 291-299.

24. Shah NP, Tran C, Lee FY, Chen P, Norris D, Sawyers CL. Overriding imatinib resistance with a novel ABL kinase inhibitor. Science. 2004;305(5682):399-401.
25. Donato NJ, Wu JY, Stapley J, et al. BCR-ABL independence and LYN kinase overexpression in chronic myelogenous leukemia cells selected for resistance to STI571. Blood. 2003;101(2): 690-698.

26. Johnson FM, Agrawal S, Burris H, et al. Phase 1 pharmacokinetic and drug-interaction study of dasatinib in patients with advanced solid tumors. Cancer. 2010;116(6):1582-1591.

27. Talpaz M, Shah NP, Kantarjian H, et al. Dasatinib in imatinibresistant Philadelphia chromosome-positive leukemias. $N$ Engl J Med. 2006;354(24):2531-2541.

28. Guilhot F, Apperley J, Kim DW, et al. Dasatinib induces significant hematologic and cytogenetic responses in patients with imatinibresistant or -intolerant chronic myeloid leukemia in accelerated phase. Blood. 2007;109(10):4143-4150.

29. Apperley JF, Cortes JE, Kim DW, et al. Dasatinib in the treatment of chronic myeloid leukemia in accelerated phase after imatinib failure: the START a trial. J Clin Oncol. 2009;27(21):3472-3479.

30. Stone RM, Kim DW, Kantarjian HM, et al. Dasatinib dose-optimization study in chronic phase chronic myeloid leukemia (CML-CP): Three-year follow-up with dasatinib $100 \mathrm{mg}$ once daily and landmark analysis of cytogenetic response and progression-free survival (PFS). J Clin Oncol (ASCO Annual Meeting Abstracts) 2009;27(15S):Abst 7007.

31. Kantarjian H, Shah NP, Hochhaus A, et al. Dasatinib versus imatinib in newly diagnosed chronic-phase chronic myeloid leukemia. $N$ Engl J Med. 2010;362(24):2260-2270.

32. Saglio G, Kim DW, Issaragrisil S, et al for ENESTnd Investigators. Nilotinib versus imatinib for newly diagnosed chronic myeloid leukemia. N Engl J Med. 2010;362(24):2251-2259.

33. Mahon F-X, Rea D, Guilhot J, et al. Discontinuation of imatinib in patients with chronic myeloid leukemia who have maintained complete molecular response: update results of the STIM Study. Blood. (ASH Annual Meeting Abstracts) 2011;118(21):Abst 603.

34. Goh, H-G, Choi S-Y, Bang J-H, et al. Discontinuation of imatinib therapy in chronic myeloid leukemia patients with sustained complete molecular response ${ }^{4.5}\left(\mathrm{CMR}^{4.5}\right)$. Blood. (ASH Annual Meeting Abstracts) 2011;118(21):Abst 2763.

35. Takahashi N, Kyo T, Maeda Y, et al. Discontinuation of imatinib in Japanese patients with chronic myeloid leukemia. Blood (ASH Annual Meeting Abstracts) 2011;118(21):Abst 3759.

36. Matsuki E, Ono Y, Sakurai M, et al. Discontinuation of imatinib in patients with CML and sustained complete molecular response (CMR) for over 2 years in the Japanese population - an interim analysis of KEIO STIM Study. Blood. (ASH Annual Meeting Abstracts) 2011;118(21): Abst 3765.

37. Rea D, Rousselot P, Nicolini F, et al. Discontinuation of dasatinib or nilotinib in chronic myeloid leukemia (CML) patients (pts) with stable undetectable Bcr-Abl transcripts: results from the French CML Group (FILMC). Blood. (ASH Annual Meeting Abstracts) 2011;118(21):Abst 604

38. Hughes TP, Hochhaus A, Branford S, et al for IRIS Investigators. Longterm prognostic significance of early molecular response to imatinib in newly diagnosed chronic myeloid leukemia: an analysis from the International Randomized Study of Interferon and STI571 (IRIS). Blood. 2010;116(19):3758-3765.

39. Hochhaus A, O'Brien SG, Guilhot F, et al for IRIS Investigators. Six-year follow-up of patients receiving imatinib for the first-line treatment of chronic myeloid leukemia. Leukemia. 2009;23(6): 1054-1061.

40. Jabbour E, Kantarjian HM, Quintas-Cardama A, et al. Impact of dose reductions and interruptions due to adverse events (AEs) on efficacy in newly diagnosed chronic myeloid leukemia in chronic phase (CML-CP) patients (pts) receiving either dasatinib (D) or imatinib (IM): analysis of the DASISION trial. Blood. (ASH Annual Meeting Abstracts) 2011;118(21):Abst 2768. 
41. Pemmaraju N, Kantarjian HM, Luthra R, et al. Results of a phase II trial of dasatinib as frontline therapy for chronic myeloid leukemia (CML) in chronic phase (CP). Blood. (ASH Annual Meeting Abstracts) 2011;118(21):Abst 1700.

42. Quintás-Cardama A, Han X, Kantarjian H, Cortes J. Tyrosine kinase inhibitor-induced platelet dysfunction in patients with chronic myeloid leukemia. Blood. 2009;114(2):261-263.

43. La Rosee P, Leitner A, Martiat P, et al. Weekend drug holiday of dasatinib in CML patients not tolerating standard dosing regimens. Reducing toxicity with maintained disease control. Blood. (ASH Annual Meeting Abstracts) 2009;114(22):Abst 1119.
44. Yassin MA, El-Ayoubi HR, Kamzoul RT. Efficacy and safety of dasatinib $50 \mathrm{mg}$ once daily dose in patients with chronic phase CML who failed imatinib. Blood. (ASH Annual Meeting Abstracts) 2011;118(21):Abst 4440.

\section{Publish your work in this journal}

Blood and Lymphatic Cancer: Targets and Therapy is an international, peer-reviewed, open access journal focusing on blood and lymphatic cancer research, identification of therapeutic targets and the optimal use of preventative and integrated treatment interventions to achieve improved outcomes, enhanced survival and quality of life for the cancer patient. The manuscript management system is completely online and includes a very quick and fair peer-review system. Visit http://www.dovepress.com/testimonials.php to read real quotes from published authors.

Submit your manuscript here: http://www.dovepress.com/blood-and-lymphatic-cancer-targets-and-therapy-journal 